Bull. Korean Math. Soc. 52 (2015), No. 2, pp. 439-452

http://dx.doi.org/10.4134/BKMS.2015.52.2.439

\title{
NEW BROWDER AND WEYL TYPE THEOREMS
}

\author{
Mohammed Berkani and Mohammed Kachad
}

\begin{abstract}
In this paper we introduce and study the new properties $(W \Pi),\left(U W \Pi_{a}\right),(U W E)$ and $(U W \Pi)$. The main goal of this paper is to study relationship between these new properties and other Weyl type theorems. Moreover, we reconsider several earlier results obtained respectively in [11], [18], [14], [1] and [13] for which we give stronger versions.
\end{abstract}

\section{Introduction}

Let $X$ be a Banach space, and let $L(X)$ be the Banach algebra of all bounded linear operators acting on $X$. For $T \in L(X)$, we will denote by $N(T)$ the null space of $T$, by $\alpha(T)$ the nullity of $T$, by $R(T)$ the range of $T$, by $\beta(T)$ its defect and by $T^{*}$ the adjoint of $T$. We will denote also by $\sigma(T)$ the spectrum of $T$ and by $\sigma_{a}(T)$ the approximate point spectrum of $T$. If the range $R(T)$ of $T$ is closed and $\alpha(T)<\infty($ resp. $\beta(T)<\infty)$, then $T$ is called an upper semi-Fredholm (resp. a lower semi-Fredholm) operator. If $T \in L(X)$ is either upper or lower semi Fredholm, then $T$ is called a semi-Fredholm operator, and the index of $T$ is defined by $\operatorname{ind}(T)=\alpha(T)-\beta(T)$. If both of $\alpha(T)$ and $\beta(T)$ are finite, then $T$ is called a Fredholm operator. An operator $T \in L(X)$ is called a Weyl operator if it is a Fredholm operator of index zero. The Weyl spectrum $\sigma_{W}(T)$ of $T$ is defined by $\sigma_{W}(T)=\{\lambda \in \mathbb{C} \mid T-\lambda I$ is not a Weyl operator $\}$.

For a bounded linear operator $T$ and a nonnegative integer $n$, define $T_{[n]}$ to be the restriction of $T$ to $R\left(T^{n}\right)$, viewed as a map from $R\left(T^{n}\right)$ into $R\left(T^{n}\right)$ (in particular $T_{[0]}=T$ ). If for some integer $n$ the range space $R\left(T^{n}\right)$ is closed and $T_{[n]}$ is an upper (resp. a lower) semi-Fredholm operator, then $T$ is called an upper (resp. a lower) semi-B-Fredholm operator. A semi-B-Fredholm operator $T$ is an upper or a lower semi-B-Fredholm operator, and in this case the index of $T$ is defined as the index of the semi-Fredholm operator $T_{[n]}$, see [12]. Moreover if $T_{[n]}$ is a Fredholm operator, then $T$ is called a B-Fredholm operator, see

Received October 10, 2013.

2010 Mathematics Subject Classification. Primary 47A53, 47A10.

Key words and phrases. property $(W \Pi)$, property $\left(U W \Pi_{a}\right)$, property $(U W \Pi)$, property $(U W E)$, Weyl-type theorems. 
[2]. An operator $T \in L(X)$ is said to be a B-Weyl operator [4], if it is a BFredholm operator of index zero. The B-Weyl spectrum $\sigma_{B W}(T)$ of $T$ is defined by $\sigma_{B W}(T)=\{\lambda \in \mathbb{C} \mid T-\lambda I$ is not a B-Weyl operator $\}$.

The ascent $a(T)$ of an operator $T$ is defined by $a(T)=\inf \left\{n \in \mathbb{N}: N\left(T^{n}\right)=\right.$ $\left.N\left(T^{n+1}\right)\right\}$, and the descent $\delta(T)$ of $T$, is defined by $\delta(T)=\inf \left\{n \in \mathbb{N}: R\left(T^{n}\right)=\right.$ $\left.R\left(T^{n+1}\right)\right\}$, with $\inf \emptyset=\infty$.

According to [15], a complex number $\lambda$ is a pole of the resolvent of $T$ if and only if $0<\max (a(T-\lambda I), \delta(T-\lambda I))<\infty$. Moreover, if this is true, then $a(T-\lambda I)=\delta(T-\lambda I)$.

Define also the set $L D(X)$ by $L D(X)=\{T \in L(X): a(T)<\infty$ and $R\left(T^{a(T)+1}\right)$ is closed $\}$ and $\sigma_{L D}(T)=\{\lambda \in \mathbb{C}: T-\lambda I \notin L D(X)\}$. Following [11], an operator $T \in L(X)$ is said to be left Drazin invertible if $T \in L D(X)$. We say that $\lambda \in \sigma_{a}(T)$ is a left pole of $T$ if $T-\lambda I \in L D(X)$, and that $\lambda \in \sigma_{a}(T)$ is a left pole of $T$ of finite rank if $\lambda$ is a left pole of $T$ and $\alpha(T-\lambda I)<\infty$.

Let $S F_{+}(X)$ be the class of all upper semi-Fredholm operators and $S F_{+}^{-}(X)$ $=\left\{T \in S F_{+}(X): \operatorname{ind}(T) \leq 0\right\}$. The upper semi-Weyl spectrum $\sigma_{S F_{+}^{-}}(T)$ of $T$ is defined by $\sigma_{S F_{+}^{-}}(T)=\left\{\lambda \in \mathbb{C}: T-\lambda I \notin S F_{+}^{-}(X)\right\}$. Similarly is defined the upper semi-B-Weyl spectrum $\sigma_{S B F_{+}^{-}}(T)$ of $T$.

In Table 1, we give a list of symbols and notations we will use:

Hereafter, the symbol $\bigsqcup$ stands for disjoint union, while iso $(A)$, means isolated points of a given subset $A$ of $\mathbb{C}$.

In this paper, we investigate the new properties $(W \Pi),\left(U W_{\Pi_{a}}\right),(U W \Pi)$, and $(U W E)$, defined below, as a continuation of our previous paper [9]. Using a new approach for the study of Weyl-type theorems based on sets differences, we begin in the second section by the study of properties $(W \Pi)$ and $\left(U W \Pi_{a}\right)$ (See Definition 2.1). Then, we give in Theorem 2.2 a fundamental result by showing that $T \in L(X)$ satisfies property $\left(U W_{\Pi_{a}}\right)$ if and only if $T$ satisfies property $(W \Pi)$ and $\sigma_{W}(T) \backslash \sigma_{S F_{+}^{-}}(T)=\left(\sigma(T) \backslash \sigma_{a}(T)\right) \sqcup\left(\Pi_{a}(T) \backslash \Pi(T)\right)$. This theorem is a typical result of the results obtained in this section and more generally in this paper. The quantity $\sigma(T) \backslash \sigma_{a}(T)$, appears to play an important role, in the comparison between Weyl-type theorems. Following the same ideas, we will give in Theorem 2.4 conditions for the equivalence of the properties $(W \Pi)$ and generalized Browder's theorem, showing also that property $(W \Pi)$ is equivalent to the property $(B b)$ introduced in [20]. A similar result gives in Theorem 2.6 conditions for the equivalence of the property $\left(U W_{\Pi_{a}}\right)$ and generalized a-Browder's theorem and it shows also that property $\left(U W_{\Pi_{a}}\right)$ and property $(S B a b))$ introduced in [10] are equivalent.

Then, in the first part of the third section, we study the properties $\left(U W_{E}\right)$ and $(U W \Pi)$ (See Definition 3.1), and we prove in Theorem 3.2 that $T$ satisfies property $\left(U W_{E}\right)$ if and only if $T$ satisfies property $\left(W_{E}\right)$ and $\sigma_{W}(T) \backslash$ $\sigma_{S F_{+}^{-}}(T)=\sigma(T) \backslash \sigma_{a}(T)$. Two other theorems linking the properties $\left(U W_{E}\right)$ and $(U W \Pi)$ to usual Weyl-type theorems are also given. In the second part 
TABle 1

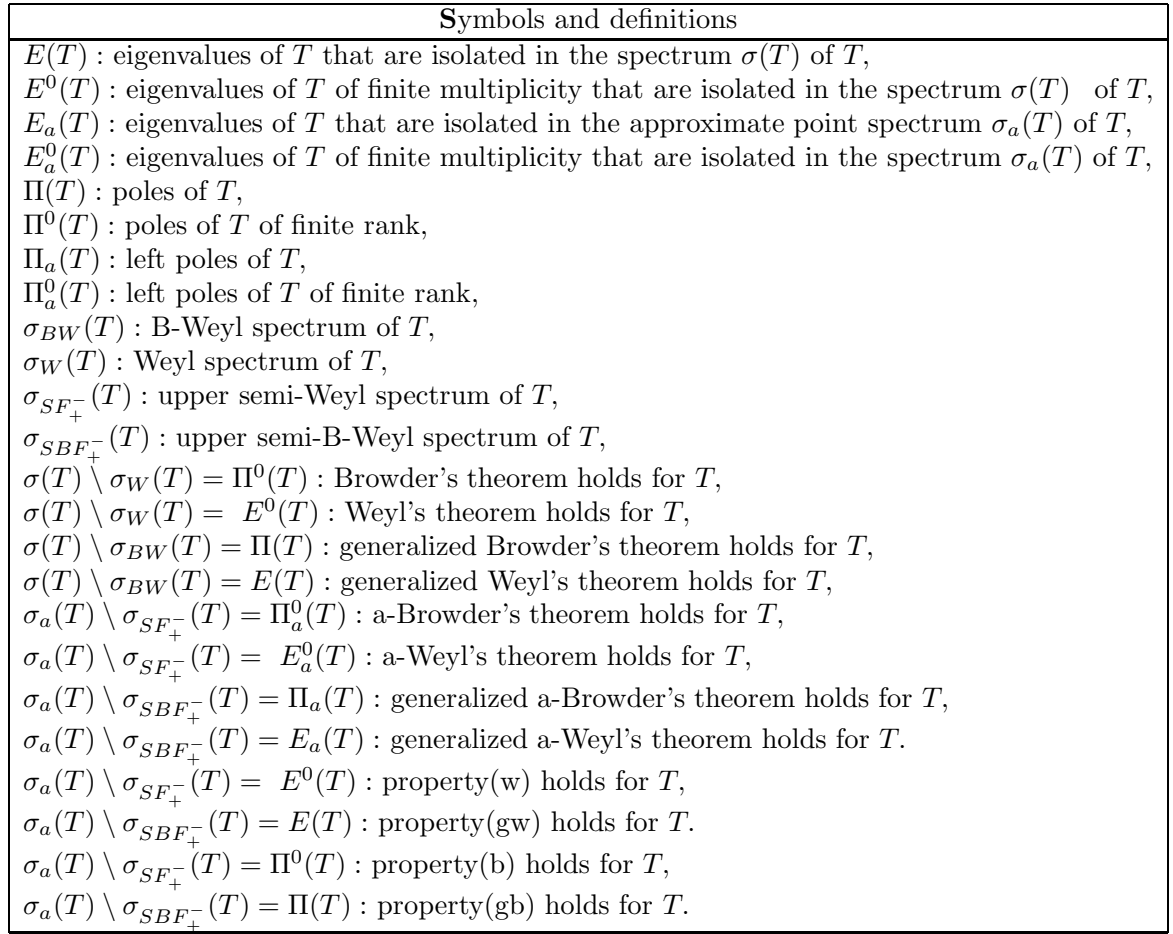

of this section, we reconsider some earlier results obtained respectively in [11], [18], [14], [1] and [13], and we give stronger versions of those theorems. As a sample of those results, we show that an operator $T \in L(X)$, satisfies generalized a-Weyl's theorem if and only if $T$ satisfies generalized Weyl's theorem, and $\sigma_{B W}(T) \backslash \sigma_{S B F_{+}^{-}}(T)=\left(\sigma(T) \backslash \sigma_{a}(T)\right) \bigsqcup\left(E_{a}(T) \backslash E(T)\right)$.

\section{Properties $(W \Pi)$ and $\left(U W \Pi_{a}\right)$}

Definition 2.1. A bounded linear operator $T \in L(X)$ is said to satisfy property $(W \Pi)$, if its spectrum is the disjoint union of its Weyl spectrum and its poles, that's $\sigma(T)=\sigma_{W}(T) \bigsqcup \Pi(T)$, and it is said to satisfy property $\left(U W \Pi_{a}\right)$, if its approximate spectrum is the disjoint union of its upper semi-Weyl spectrum and its left poles, that's $\sigma_{a}(T)=\sigma_{S F_{+}^{-}}(T) \bigsqcup \Pi_{a}(T)$.

Theorem 2.2. Suppose that $T \in L(X)$. Then the following statements are equivalent:

(i) T satisfies property $\left(U W \Pi_{a}\right)$; 
(ii) $T$ satisfies property $(W \Pi)$ and

$$
\sigma_{W}(T) \backslash \sigma_{S F_{+}^{-}}(T)=\left(\sigma(T) \backslash \sigma_{a}(T)\right) \bigsqcup\left(\Pi_{a}(T) \backslash \Pi(T)\right) .
$$

Proof. (i) $\Rightarrow$ (ii) Suppose that $T$ satisfies property $\left(U W \Pi_{a}\right)$, that's $\sigma_{a}(T)=$ $\sigma_{S F_{+}^{-}}(T) \bigsqcup \Pi_{a}(T)$. Let us prove that

$$
\sigma_{W}(T) \backslash \sigma_{S F_{+}^{-}}(T)=\left(\sigma(T) \backslash \sigma_{a}(T)\right) \bigsqcup\left(\Pi_{a}(T) \backslash \Pi(T)\right) .
$$

If $\lambda \in \sigma(T) \backslash \sigma_{a}(T)$, then $\alpha(T-\lambda I)=0$ and $R(T-\lambda I)$ is closed in the Banach space $X$. This implies that $\lambda \notin \sigma_{S F_{+}^{-}}(T)$, and we also have $\lambda \in \sigma_{W}(T)$. Suppose to the contrary, that's $\lambda \notin \sigma_{W}(T)$, then $\alpha(T-\lambda I)=\beta(T-\lambda I)=0$. Hence $\lambda \notin \sigma(T)$, and we get the desired contradiction. So $\lambda \in \sigma_{W}(T) \backslash \sigma_{S F_{+}^{-}}(T)$. Now suppose that $\lambda \in \Pi_{a}(T) \backslash \Pi(T)$. Since $T$ satisfies property $\left(U W \Pi_{a}\right)$, it follows that $\lambda \notin \sigma_{S F_{+}^{-}}(T)$. We have also $\lambda \in \sigma_{W}(T)$. Indeed, if $\lambda \notin \sigma_{W}(T)$, then by the uniqueness of the index of a semi-B-Fredholm operator [12, Proposition 2.1], we have $\alpha(T-\lambda I)=\beta(T-\lambda I)$ and $a(T-\lambda I)<+\infty$. Hence $\delta(T-\lambda I)<+\infty$ and $\lambda \in \Pi(T)$, which is a contradiction. So $\lambda \in \sigma_{W}(T) \backslash \sigma_{S F_{+}^{-}}(T)$. It is clear that $\left(\sigma(T) \backslash \sigma_{a}(T)\right) \cap\left(\Pi_{a}(T) \backslash \Pi(T)\right)=\emptyset$. Consequently, we have $(\sigma(T) \backslash$ $\left.\sigma_{a}(T)\right) \bigsqcup\left(\Pi_{a}(T) \backslash \Pi(T)\right) \subseteq \sigma_{W}(T) \backslash \sigma_{S F_{+}^{-}}(T)$. To show the reverse inclusion, suppose that $\lambda \in \sigma_{W}(T) \backslash \sigma_{S F_{+}^{-}}(T)$. Since $T$ satisfies property $\left(U W \Pi_{a}\right)$, then $a(T-\lambda I)<+\infty, \alpha(T-\lambda I)<+\infty$ and $R(T-\lambda I)$ is closed. Hence $\delta(T-\lambda I)=$ $+\infty$, because otherwise we would have $\lambda \notin \sigma_{W}(T)$. So if $\alpha(T-\lambda I)=0$, $\lambda \in \sigma(T) \backslash \sigma_{a}(T)$, and if $\alpha(T-\lambda I)>0$, then $\lambda \in \Pi_{a}(T) \backslash \Pi(T)$. Therefore $\lambda \in\left(\sigma(T) \backslash \sigma_{a}(T)\right) \bigsqcup\left(\Pi_{a}(T) \backslash \Pi(T)\right)$, and we have $\sigma_{W}(T) \backslash \sigma_{S F_{+}^{-}}(T)=(\sigma(T) \backslash$ $\left.\sigma_{a}(T)\right) \bigsqcup\left(\Pi_{a}(T) \backslash \Pi(T)\right)$, as required.

Now let us prove that $T$ satisfies property $(W \Pi)$. As we have $\sigma(T)=(\sigma(T) \backslash$ $\left.\sigma_{a}(T)\right) \bigsqcup \sigma_{a}(T)$, and since $T$ satisfies property $\left(U W_{\Pi_{a}}\right)$, then $\sigma(T)=(\sigma(T) \backslash$ $\left.\sigma_{a}(T)\right) \bigsqcup \sigma_{S F_{+}^{-}}(T) \bigsqcup \Pi_{a}(T)$. Hence $\sigma(T)=\sigma_{S F_{+}^{-}}(T) \bigsqcup\left(\sigma(T) \backslash \sigma_{a}(T)\right) \bigsqcup\left(\Pi_{a}(T) \backslash\right.$ $\Pi(T)) \bigsqcup \Pi(T)^{+}$. As we know that $\sigma_{W}(T) \backslash \sigma_{S F_{+}^{-}}(T)=\left(\sigma(T) \backslash \sigma_{a}(T)\right) \bigsqcup\left(\Pi_{a}(T) \backslash\right.$ $\Pi(T))$, then $\sigma(T)=\sigma_{W}(T) \bigsqcup \Pi(T)$ and $T$ satisfies property $(W \Pi)$.

(ii) $\Rightarrow$ (i) As $\sigma_{a}(T)=\sigma_{L D}(T) \bigsqcup \Pi_{a}(T)$, it is sufficient to prove that $\sigma_{L D}(T)$ $=\sigma_{S F_{+}^{-}}(T)$. If $\lambda \in \sigma_{L D}(T)$, since $\sigma_{W}(T) \backslash \sigma_{S F_{+}^{-}}(T)=\left(\sigma(T) \backslash \sigma_{a}(T)\right) \bigsqcup\left(\Pi_{a}(T) \backslash\right.$ $\Pi(T))$, then $\lambda \notin \sigma_{W}(T) \backslash \sigma_{S F_{+}^{-}}(T)$. As $T$ satisfies property $(W \Pi)$, then $\sigma(T)=$ $\sigma_{W}(T) \backslash \sigma_{S F_{+}^{-}}(T) \bigsqcup \sigma_{S F_{+}^{-}}(T) \bigsqcup \Pi(T)$, and so $\lambda \in \sigma_{S F_{+}^{-}}(T)$. It is clear that $\sigma_{S F_{+}^{-}}(T) \subset \sigma_{L D}(T)$, then $\sigma_{a}(T)=\sigma_{S F_{+}^{-}}(T) \bigsqcup \Pi_{a}(T)$ and $T$ satisfies property $\left(U W \Pi_{a}\right)$.

Example 2.3. In general, we cannot expect that property $\left(U W \Pi_{a}\right)$ holds for an operator satisfying property $(W \Pi)$. For this, let $T$ be the operator defined on the Banach space $\ell^{2}(\mathbb{N}) \oplus \ell^{2}(\mathbb{N})$ by $T=R \oplus 0$, where $R$ is the unilateral right shift operator. Then we have $\sigma_{a}(T)=\sigma_{S F_{+}^{-}}(T)=C(0,1) \cup\{0\}, \sigma_{S B F_{+}^{-}}(T)=C(0,1)$ 
and $\Pi_{a}(T)=\{0\}$. Moreover, we have $\sigma(T)=\sigma_{W}(T)=\sigma_{B W}(T)=D(0,1)$ and $\Pi(T)=\emptyset$. So $T$ satisfies property $(W \Pi)$, but $T$ does not satisfies property $\left(U W \Pi_{a}\right)$.

An operator $T \in L(X)$ satisfies property $(B b)([20])$ if

$$
\sigma(T)=\sigma_{B W}(T) \bigsqcup \Pi^{0}(T) .
$$

In the following theorem we establish a relationship between property $\left(W_{\Pi}\right)$, generalized Browder's theorem and property $(B b)$.

Theorem 2.4. Suppose that $T \in L(X)$. Then the following statements are equivalent:

(i) $T$ satisfies property $(W \Pi)$;

(ii) $T$ satisfies generalized Browder's theorem and $\sigma_{W}(T) \backslash \sigma_{B W}(T)=$ $\Pi(T) \backslash \Pi^{0}(T)=\emptyset$

(iii) $T$ satisfies property $(B b)$.

Proof. (i) $\Rightarrow$ (ii) Suppose that $T$ satisfies property $(W \Pi)$, that's

$$
\sigma(T)=\sigma_{W}(T) \bigsqcup \Pi(T) .
$$

Let $\lambda \in \sigma(T)$ be given. If $\lambda \in \sigma_{W}(T)$, then $\lambda \notin \Pi(T)$, and $\lambda \in \sigma_{B W}(T)$. Indeed, if $\lambda \notin \sigma_{B W}(T)$, then $T-\lambda I$ is a B-Weyl operator. From [4, Theorem 2.4], if $\eta$ is small enough and $|\eta|>0$, then $T-\lambda I-\eta I$ is a Weyl operator. Hence $\lambda$ is an isolated point in $\sigma_{W}(T)$. As $\sigma(T)=\sigma_{W}(T) \bigsqcup \Pi(T)$, then $\lambda$ is an isolated point in $\sigma(T)$. From [5, Theorem 2.3], $\lambda \in \Pi(T)$, and this is a contradiction. As we have always $\sigma_{B W}(T) \subseteq \sigma_{W}(T)$, then $\sigma_{B W}(T)=\sigma_{W}(T)$. In the same way, if $\lambda \in \Pi(T)$, as $\sigma(T)=\sigma_{W}(T) \bigsqcup \Pi(T)$, then $\lambda \notin \sigma_{W}(T)$. Hence $\alpha(T-\lambda I)<\infty$, and so $\lambda \in \Pi^{0}(T)$. As we have always $\Pi^{0}(T) \subset \Pi(T)$, then $\Pi(T)=\Pi^{0}(T)$. Consequently, $T$ satisfies generalized Browder's theorem and $\sigma_{B W}(T) \backslash \sigma_{W}(T)=\Pi(T) \backslash \Pi^{0}(T)=\emptyset$.

(ii) $\Rightarrow$ (iii) Obvious.

(iii) $\Rightarrow$ (i) Assume that $T$ satisfies property $(B b)$, then

$$
\sigma(T)=\sigma_{B W}(T) \bigsqcup \Pi^{0}(T) .
$$

As we have always $\sigma_{B W}(T) \subset \sigma_{W}(T)$, then $\sigma(T)=\sigma_{W}(T) \bigsqcup \Pi^{0}(T)$. On another way, if $\lambda \in \Pi(T)$, then $\lambda \notin \sigma_{B W}(T)$. Hence $\Pi^{0}(T)=\Pi(T)$. So $\sigma(T)=\sigma_{W}(T) \bigsqcup \Pi(T)$ and $T$ satisfies property $(W \Pi)$.

Remark 2.5. From Theorem 2.4, if $T \in L(X)$ satisfies property (Wח), then it satisfies generalized Browder's theorem. However, the converse is not true in general as seen by the following example:

Let $X=\ell^{2}(\mathbb{N})$, let $B=\left\{e_{i} \mid e_{i}=\left(\delta_{i}^{j}\right)_{j \in \mathbb{N}}, i \in \mathbb{N}\right\}$ be the canonical basis of $\ell^{2}(\mathbb{N})$. Let $E$ be the subspace of $\ell^{2}(\mathbb{N})$ generated by the set $\left\{e_{i} \mid 1 \leq i \leq n\right\}$. Let $\mathrm{P}$ be the orthogonal projection on $E$. Then $\sigma(P)=\{0,1\}, \sigma_{W}(\bar{P})=\{0\}$, $\sigma_{B W}(P)=\emptyset$ and $\Pi(P)=\{0,1\}$. So $P$ satisfies generalized Browder's theorem. But $P$ does not satisfy property $(W \Pi)$, since $\sigma_{W}(P) \cap \Pi(P) \neq \emptyset$. 
An operator $T \in L(X)$ satisfies property $(S B a b)$ (see [10]) if $\sigma_{a}(T)=$ $\sigma_{S B F_{+}^{-}}(T) \bigsqcup \Pi_{a}^{0}(T)$. In the following theorem we establish a relationship between property $\left(U W \Pi_{a}\right)$, generalized a-Browder's theorem and property $(S B a b)$.

Theorem 2.6. Suppose that $T \in L(X)$. Then the following statements are equivalent:

(i) $T$ satisfies property $\left(U W \Pi_{a}\right)$;

(ii) $T$ satisfies generalized a-Browder's theorem and $\sigma_{S F_{+}^{-}}(T) \backslash \sigma_{S B F_{+}^{-}}(T)=$ $\Pi_{a}(T) \backslash \Pi_{a}^{0}(T)=\emptyset ;$

(iii) $T$ satisfies property $(S B a b)$.

Proof. (i) $\Rightarrow$ (ii) Suppose that $T$ satisfies property $\left(U W \Pi_{a}\right)$, that's

$$
\sigma_{a}(T)=\sigma_{S F_{+}^{-}}(T) \bigsqcup \Pi_{a}(T) .
$$

Let $\lambda \in \sigma_{a}(T)$ be given. If $\lambda \in \sigma_{S F_{+}^{-}}(T)$, then $\lambda \notin \Pi_{a}(T)$. We assert that $\lambda \in$ $\sigma_{S B F_{+}^{-}}(T)$. Indeed, if $\lambda \notin \sigma_{S B F_{+}^{-}}(T)$, then $T-\lambda I$ is an upper semi-B-Fredholm operator. From [12, Corollary 3.2], if $\eta$ is small enough and $|\eta|>0$, then $T-\lambda I-\eta I$ is an upper semi-Fredholm operator. Hence $\lambda$ is an isolated point in $\sigma_{S F_{+}^{-}}(T)$. As $\sigma_{a}(T)=\sigma_{S F_{+}^{-}}(T) \bigsqcup \Pi_{a}(T)$, then $\lambda$ is an isolated point in $\sigma_{a}(T)$. From [9, Theorem 2.8], it follows that $\lambda \in \Pi_{a}(T)$, which is a contradiction. As we have always $\sigma_{S B F_{+}^{-}}(T) \subseteq \sigma_{S F_{+}^{-}}(T)$, then $\sigma_{S B F_{+}^{-}}(T)=\sigma_{S F_{+}^{-}}(T)$. In the same way, if $\lambda \in \Pi_{a}(T)$, as $\sigma_{a}(T)=\sigma_{S F_{+}^{-}}(T) \bigsqcup \Pi_{a}(T)$, then $\lambda \notin \sigma_{S F_{+}^{-}}(T)$. Hence $\alpha(T-\lambda I)<\infty$, and so $\lambda \in \Pi_{a}^{0}(T)$. As we have always $\Pi_{a}^{0}(T) \subset \Pi_{a}(T)$, then $\Pi_{a}(T)=\Pi_{a}^{0}(T)$. Consequently, $T$ satisfies generalized a-Browder's theorem and $\sigma_{S F_{+}^{-}}(T) \backslash \sigma_{S B F_{+}^{-}}(T)=\Pi_{a}(T) \backslash \Pi_{a}^{0}(T)=\emptyset$.

(ii) $\Rightarrow$ (iii) Obvious.

(iii) $\Rightarrow$ (i) Assume that $T$ satisfies property $(S B a b)$, that's

$$
\sigma_{a}(T)=\sigma_{S B F_{+}^{-}}(T) \bigsqcup \Pi_{a}^{0}(T) .
$$

Then, it's easily seen that $\sigma_{S B F_{+}^{-}}(T)=\sigma_{S F_{+}^{-}}(T)$. As $\sigma_{a}(T)=\sigma_{L D}(T) \bigsqcup \Pi_{a}(T)$, $\sigma_{S F_{+}^{-}}(T) \subseteq \sigma_{L D}(T)$, and $\Pi_{a}^{0}(T) \subset \Pi_{a}(T)$, then $\sigma_{a}(T)=\sigma_{S F_{+}^{-}}(T) \bigsqcup \Pi_{a}(T)$. Therefore $T$ satisfies property $\left(U W \Pi_{a}\right)$.

The following example shows that there exist operators satisfying generalized a-Browder's theorem but not property $\left(U W \Pi_{a}\right)$.

Example 2.7. On $\ell^{2}(\mathbb{N})$, let $T$ be defined by: $T\left(x_{1}, x_{2}, x_{3}, \ldots\right)=\left(0, \frac{1}{2} x_{1}, 0,0\right.$, $\ldots)$. Then $\sigma_{a}(T)=\sigma_{S F_{+}^{-}}(T)=\{0\}$ and $\Pi_{a}(T)=\{0\}$. As $T$ is nilpotent, then $\sigma_{S B F_{+}^{-}}(T)=\emptyset$. So $T$ satisfies generalized a-Browder's theorem, but $T$ does not satisfy property $\left(U W \Pi_{a}\right)$. 
An operator $T \in L(X)$ satisfies property $(W E)$ (resp. $\left(U W_{E_{a}}\right)$ ), if $\sigma(T)=$ $\sigma_{W}(T) \bigsqcup E(T)\left(\right.$ resp. $\left.\sigma_{a}(T)=\sigma_{S F_{+}^{-}}(T) \bigsqcup E_{a}(T)\right)$ (see [9] for more details).

Theorem 2.8. Let $T \in L(X)$. Then the following statements are equivalent:

(i) $T$ satisfies property $\left(W_{E}\right)$;

(ii) $T$ satisfies property $(W \Pi)$, and $E(T)=\Pi(T)$.

Proof. (i) $\Rightarrow\left(\right.$ ii) Suppose that $T$ satisfies property $\left(W_{E}\right)$, that's

$$
\sigma(T)=\sigma_{W}(T) \bigsqcup E(T) .
$$

From [9, Remark 2.3], we have $\Pi^{0}(T)=\Pi(T)=E(T)$. Then it follows that $T$ satisfies property $(W \Pi)$, and $\Pi(T)=E(T)$.

(ii) $\Rightarrow$ (i) Since $T$ satisfies property $(W \Pi)$, then $\sigma(T)=\sigma_{W}(T) \bigsqcup \Pi(T)$. As by assumption $\Pi(T)=E(T)$, so $\sigma(T)=\sigma_{W}(T) \bigsqcup E(T)$, and $T$ satisfies property $\left(W_{E}\right)$.

Remark 2.9. From Theorem 2.8, if $T \in L(X)$, satisfies property $\left(W_{E}\right)$, then it satisfies $\left(W_{\Pi}\right)$. However the converse is not true in general. For this, let $Q \in L\left(\ell^{2}(\mathbb{N})\right)$ defined by: $Q\left(x_{0}, x_{1}, \ldots\right)=\left(\frac{1}{2} x_{2}, \frac{1}{3} x_{3}, \ldots\right)$ for $\left(x_{n}\right)_{n} \in \ell^{2}(\mathbb{N})$. Consider the operator $T$ defined on $\left.\ell^{2}(\mathbb{N}) \oplus \ell^{2}(\mathbb{N})\right)$ by $T=Q \oplus 0$, then $\sigma(T)=$ $\sigma_{W}(T)=\{0\}, E(T)=\{0\}$ and $E^{0}(T)=\Pi(T)=\Pi^{0}(T)=\emptyset$. So $T$ satisfies property $\left(W_{\Pi}\right)$, but does not satisfy property $\left(W_{E}\right)$.

Theorem 2.10. Let $T \in L(X)$. Then the following statements are equivalent:

(i) $T$ satisfies property $\left(U W E_{a}\right)$;

(ii) $T$ satisfies property $\left(U W_{\Pi_{a}}\right)$, and $\Pi_{a}(T)=E_{a}(T)$.

Proof. (i) $\Rightarrow$ (ii) Suppose that $T$ satisfies property $\left(U W_{E_{a}}\right)$. Then from $[9$, Theorem 3.2], and [9, Remark 3.4] $T$ satisfies generalized a-Weyl's theorem and $\Pi_{a}^{0}(T)=\Pi_{a}(T)=E_{a}^{0}(T)=E_{a}(T)$. Thus $T$ satisfies property $\left(U W \Pi_{a}\right)$, and $\Pi_{a}(T)=E_{a}(T)$.

(ii) $\Rightarrow$ (i) Obvious.

Theorem 2.8 and Theorem 2.10 are similar respectively to [6, Theorem 2.9] and [6, Theorem 2.10], in which conditions for the equivalence of generalized Weyl's (resp. generalized a-Weyl's) theorem and Weyl's (resp. a-Weyl's) theorem are given.

In the next theorem, and similarly to Theorem 2.2 , we give precise conditions under which the properties $\left(U W_{E_{a}}\right)$ and $\left(W_{E}\right)$ are equivalent.

Theorem 2.11. Suppose that $T \in L(X)$. Then the following statements are equivalent:

(i) $T$ satisfies property $\left(U W_{E_{a}}\right)$;

(ii) $T$ satisfies property $\left(W_{E}\right)$, and

$$
\sigma_{W}(T) \backslash \sigma_{S F_{+}^{-}}(T)=\left(\sigma(T) \backslash \sigma_{a}(T)\right) \bigsqcup\left(E_{a}(T) \backslash E(T)\right) .
$$


Proof. Suppose that $T$ satisfies property $\left(U W E_{a}\right)$. Using Theorem 2.10, we see that $T$ satisfies property $\left(U W \Pi_{a}\right)$, and $\Pi_{a}(T)=E_{a}(T)$. Then from Theorem 2.2, $T$ satisfies property $(W \Pi)$ and $\sigma_{W}(T) \backslash \sigma_{S F_{+}^{-}}(T)=\left(\sigma(T) \backslash \sigma_{a}(T)\right) \bigsqcup\left(\Pi_{a}(T) \backslash\right.$ $\Pi(T))$. Let $\lambda \in E(T)$, then $\lambda \in E_{a}(T)$. As $\Pi_{a}^{+}(T)=E_{a}(T)$, then $\lambda \in \Pi_{a}(T)$ and $T-\lambda I$ is left Drazin invertible. Since $\lambda$ is isolated in $\sigma(T)$, then from $[12$, Theorem 3.1], $\lambda \in \Pi(T)$. As we have always $\Pi(T) \subset E(T)$, then $\Pi(T)=E(T)$. As $T$ satisfies $\left(W_{\Pi}\right)$ and $\Pi(T)=E(T)$, then from Theorem 2.8, $T$ satisfies property $\left(W_{E}\right)$. Moreover, taking into account that $\Pi(T)=E(T)$ and $\Pi_{a}(T)=$ $E_{a}(T)$, we see that $\sigma_{W}(T) \backslash \sigma_{S F_{+}^{-}}(T)=\left(\sigma(T) \backslash \sigma_{a}(T)\right) \bigsqcup\left(E_{a}(T) \backslash E(T)\right)$.

Conversely, as $T$ satisfies property $\left(W_{E}\right)$, then

$$
\sigma(T)=\left(\sigma_{W}(T) \backslash \sigma_{S F_{+}^{-}}(T)\right) \bigsqcup \sigma_{S F_{+}^{-}}(T) \bigsqcup E(T) .
$$

Since by assumption we have $\sigma_{W}(T) \backslash \sigma_{S F_{+}^{-}}(T)=\left(\sigma(T) \backslash \sigma_{a}(T)\right) \bigsqcup\left(E_{a}(T) \backslash\right.$ $E(T))$, it follows that

$$
\sigma(T)=\left(\sigma(T) \backslash \sigma_{a}(T)\right) \bigsqcup \sigma_{S F_{+}^{-}}(T) \bigsqcup\left(E_{a}(T) \backslash E(T)\right) \bigsqcup E(T) .
$$

Therefore, we have $\sigma_{a}(T)=\sigma_{S F_{+}^{-}}(T) \bigsqcup E_{a}(T)$, and $T$ satisfies property $\left(U W_{E_{a}}\right)$.

\section{Properties $\left(U W_{E}\right)$ and $\left(U W_{\Pi}\right)$}

Definition 3.1. A Bounded linear operator $T \in L(X)$ is said to satisfy property $(U W E)$ (resp. $(U W \Pi))$ if its approximate spectrum is the disjoint union of its upper semi-Weyl spectrum and its isolated eigenvalues (resp. of its upper semi-Weyl spectrum and its poles), that's $\sigma_{a}(T)=\sigma_{S F_{+}^{-}}(T) \bigsqcup E(T)$ (resp. $\left.\sigma_{a}(T)=\sigma_{S F_{+}^{-}}(T) \bigsqcup \Pi(T)\right)$.

We start by the following result giving a characterization of operators satisfying property $(U W E)$.

Theorem 3.2. Let $T \in L(X)$. Then the following statements are equivalent:

(i) $T$ satisfies property $(U W E)$;

(ii) $T$ satisfies property $(W E)$, and $\sigma_{W}(T) \backslash \sigma_{S F_{+}^{-}}(T)=\sigma(T) \backslash \sigma_{a}(T)$.

Proof. Suppose that $T$ satisfies property $\left(U W_{E}\right)$. Let $\lambda \in \sigma_{W}(T) \backslash \sigma_{S F_{+}^{-}}(T)$ be given. Then $\lambda \in \sigma(T), \alpha(T-\lambda)<\infty$ and $R(T-\lambda I)$ is closed. If $\alpha\left(T^{+}-\lambda\right)>$ 0 , then $\lambda \in \sigma_{a}(T)$. As $T$ satisfies property $(U W E)$, then $\lambda \in E(T)$, and so $\lambda$ is an isolated point in $\sigma(T)$. From [12, Theorem 3.1 and Corollary 3.2], we get that $\operatorname{ind}(T-\lambda I)=0$. But this is a contradiction, since $\lambda \in \sigma_{W}(T)$. Consequently $\alpha(T-\lambda)=0$, and $\lambda \in \sigma(T) \backslash \sigma_{a}(T)$. Now if $\lambda \in \sigma(T) \backslash \sigma_{a}(T)$, then $\lambda \notin \sigma_{S F_{+}^{-}}(T)$. Assume that $T-\lambda I$ is a Weyl operator. Since $\alpha(T-\lambda)=0$ then $T-\lambda I$ would be invertible. But this is impossible, since $\lambda \in \sigma(T)$. So $\sigma_{W}(T) \backslash \sigma_{S F_{+}^{-}}(T)=\sigma(T) \backslash \sigma_{a}(T)$. As $\sigma(T)=\left(\sigma(T) \backslash \sigma_{a}(T)\right) \bigsqcup \sigma_{a}(T)$, and since 
$T$ satisfies property $(U W E)$, then $\sigma(T)=\left(\sigma(T) \backslash \sigma_{a}(T)\right) \bigsqcup \sigma_{S F_{+}^{-}}(T) \bigsqcup E(T)$. Hence, $\sigma(T)=\sigma_{W}(T) \bigsqcup E(T)$ and $T$ satisfies property $\left(W_{E}\right)$.

Conversely, suppose that $T$ satisfies property $\left(W_{E}\right)$ and $\sigma_{W}(T) \backslash \sigma_{S F_{+}^{-}}(T)=$ $\sigma(T) \backslash \sigma_{a}(T)$. Then $\sigma(T)=\left(\sigma_{W}(T) \backslash \sigma_{S F_{+}^{-}}(T)\right) \sqcup \sigma_{S F_{+}^{-}}(T) \sqcup E(T)$. Therefore, $\sigma_{a}(T)=\sigma_{S F_{+}^{-}}(T) \bigsqcup E(T)$, and so $T$ satisfies property $\left(U W_{E}\right)$.

The following examples show that property $\left(U W_{E_{a}}\right)$ and property $(U W E)$ are not related to each other.

Example 3.3. Let $R \in L\left(\ell^{2}(\mathbb{N})\right)$ be the unilateral right shift and let $U$ be defined on $\ell^{2}(\mathbb{N})$ by $U\left(x_{1}, x_{2}, \ldots\right)=\left(0, x_{2}, x_{3}, \ldots\right),\left(x_{n}\right) \in \ell^{2}(\mathbb{N})$. If $T=$ $R \oplus U$, then $\sigma(T)=\sigma_{W}(T)=\sigma_{B W}(T)=D(0,1)$, the closed unit disc in $\mathbb{C}$, iso $(\sigma(T))=E(T)=\emptyset$. Moreover, we have $\sigma_{a}(T)=C(0,1) \cup\{0\}$, where $C(0,1)$ is unit circle of $\mathbb{C}, \sigma_{S F_{+}^{-}}(T)=\sigma_{S B F_{+}^{-}}=C(0,1)$. This implies that $\sigma_{a}(T) \backslash \sigma_{S F_{+}^{-}}(T)=\{0\}$ and $E_{a}^{+}(T)=\{0\}$. Consequently $T$ satisfies property $\left(U W E_{a}\right)$, but $T$ does not satisfy property $(U W E)$.

Example 3.4. Let $T$ be defined on the Banach space $\ell^{2}(\mathbb{N}) \oplus \ell^{2}(\mathbb{N})$ by $T=R \oplus$ 0 , where $R$ is the unilateral right shift operator. We have $\sigma_{a}(T)=\sigma_{S F_{+}^{-}}(T)=$ $C(0,1) \cup\{0\}, \sigma_{S B F_{+}^{-}}(T)=C(0,1)$ and $E_{a}(T)=\{0\}$. Moreover, we have $\sigma(T)=$ $\sigma_{W}(T)=\sigma_{B W}(T)=D(0,1)$ and $E(T)=\emptyset$. So $T$ satisfies property $\left(U W_{E}\right)$, but $T$ does not satisfies property $\left(U W E_{a}\right)$.

Similarly to Theorem 3.2 , we give, without proof, in the following theorem a characterization of operators satisfying property $(U W \Pi)$.

Theorem 3.5. Let $T \in L(X)$. Then the following statements are equivalent:

(i) $T$ satisfies property $(U W \Pi)$;

(ii) $T$ satisfies property $(W \Pi)$, and $\sigma_{W}(T) \backslash \sigma_{S F_{+}^{-}}(T)=\sigma(T) \backslash \sigma_{a}(T)$.

Remark 3.6. If $T$ satisfies property $\left(U W_{E}\right)$, then $E(T)=E^{0}(T)$, and if $T$ satisfies property $(U W \Pi)$, then $\Pi(T)=\Pi^{0}(T)$.

Theorem 3.7. Let $T \in L(X)$ such that $T$ satisfies property $(U W E)$. Then $T$ satisfies generalized a-Weyl's theorem if and only if $\sigma_{S F_{+}^{-}}(T) \backslash \sigma_{S B F_{+}^{-}}(T)=$ $E_{a}(T) \backslash E(T)$.

Proof. Assume that $T$ satisfies property $(U W E)$, then $\sigma_{a}(T)=\sigma_{S F_{+}^{-}}(T) \bigsqcup E(T)$. Therefore $\sigma_{a}(T)=\sigma_{S B F_{+}^{-}}(T) \bigsqcup\left(\sigma_{S F_{+}^{-}}(T) \backslash \sigma_{S B F_{+}^{-}}(T)\right) \bigsqcup E(T)$. If $T$ satisfies generalized a-Weyl's theorem, then $\sigma_{a}(T)=\sigma_{S B F_{+}^{-}}(T) \bigsqcup E_{a}(T)$. Hence $\left(\sigma_{S F_{+}^{-}}(T) \backslash \sigma_{S B F_{+}^{-}}(T)\right) \sqcup E(T)=E_{a}(T)$, and so $\sigma_{S F_{+}^{-}}(T) \backslash \sigma_{S B F_{+}^{-}}(T)=E_{a}(T) \backslash$ $E(T)$.

Conversely, assume that $T$ satisfies property $(U W E)$, and

$$
\sigma_{S F_{+}^{-}}(T) \backslash \sigma_{S B F_{+}^{-}}(T)=E_{a}(T) \backslash E(T) .
$$


Then

$$
\begin{aligned}
\sigma_{a}(T) & =\sigma_{S F_{+}^{-}}(T) \bigsqcup E(T)=\left(\sigma_{S F_{+}^{-}}(T) \backslash \sigma_{S B F_{+}^{-}}(T)\right) \bigsqcup \sigma_{S B F_{+}^{-}}(T) \bigsqcup E(T) \\
& =\sigma_{S B F_{+}^{-}}(T) \bigsqcup E_{a}(T) .
\end{aligned}
$$

So $T$ satisfies generalized a-Weyl's theorem.

Similarly to Theorem 3.7, we give now a result linking property $(U W \Pi)$ and generalized a-Browder's theorem.

Theorem 3.8. Let $T \in L(X)$ such that $T$ satisfies property $(U W \Pi)$. Then $T$ satisfies generalized a-Browder's theorem if and only if $\sigma_{S F_{+}^{-}}(T) \backslash \sigma_{S B F_{+}^{-}}(T)=$ $\Pi_{a}(T) \backslash \Pi(T)$.

The approach based on set differences used in the previous results, appears to be an efficient tool in studying relationship between Weyl-type theorems. Following this path, we reconsider hereunder several results obtained respectively in [11, Theorem 3.7], [11, Theorem 3.8], [18, Corollary 2.5], [14, Theorem $3.9]$, [1, Theorem 2.3] and [13, Theorem 2.3], for which we give stronger versions. We will give the proof of the first theorem, avoiding redundancy, we give the others without proof. For the definitions of the different properties used hereunder, we refer the reader to Table 1.

In [11, Theorem 3.7], it had been proved that condition (i) of the following theorem implies the first part of its second condition (ii). Here, we obtain an equivalence of the statements (i) and (ii).

Theorem 3.9. Suppose that $T \in L(X)$. Then the following statements are equivalent:

(i) T satisfies generalized a-Weyl's theorem;

(ii) $T$ satisfies generalized Weyl's theorem, and $\sigma_{B W}(T) \backslash \sigma_{S B F_{+}^{-}}(T)=$ $\left(\sigma(T) \backslash \sigma_{a}(T)\right) \bigsqcup\left(E_{a}(T) \backslash E(T)\right)$.

Proof. Assume that $T$ satisfies generalized a-Weyl's theorem. Then $\sigma_{a}(T)=$ $\sigma_{S B F_{+}^{-}}(T) \bigsqcup E_{a}(T)$. Let $\lambda \in \sigma_{B W}(T) \backslash \sigma_{S B F_{+}^{-}}(T)$. If $\lambda \in \sigma_{a}(T)$, then $\lambda \in$ $E_{a}(T)$. In this case, if $\lambda \in E(T)$, then from [12, Theorem 3.1], we will have $T-\lambda I$ a B-Weyl operator. But this is impossible since $\lambda \in \sigma_{B W}(T)$. Therefore $\lambda \in E_{a}(T) \backslash E(T)$. If $\lambda \notin \sigma_{a}(T)$, then $\lambda \in \sigma(T) \backslash \sigma_{a}(T)$. Consequently $\sigma_{B W}(T) \backslash$ $\sigma_{S B F_{+}^{-}}(T) \subset\left(\sigma(T) \backslash \sigma_{a}(T)\right) \bigsqcup\left(E_{a}(T) \backslash E(T)\right)$.

Now if $\lambda \in \sigma(T) \backslash \sigma_{a}(T)$, then $T-\lambda I$ is injective with closed range. Hence $\lambda \in \sigma_{B W}(T)$ because, otherwise we would have $T-\lambda I$ invertible, but this is impossible, since $\lambda \in \sigma(T)$. So $\lambda \in \sigma_{B W}(T) \backslash \sigma_{S B F_{+}^{-}}(T)$. Similarly, if $\lambda \in$ $E_{a}(T) \backslash E(T)$, as $T$ satisfies generalized a-Weyl's theorem, then $\lambda \notin \sigma_{S B F_{+}^{-}}(T)$. Since $\lambda \notin E(T)$, necessarily $\lambda \in \sigma_{B W}(T)$. Indeed, if $\lambda \notin \sigma_{B W}(T)$, then $T-\lambda I$ is a B-Weyl operator. From [4, Theorem 2.4], if $\eta$ is small enough and $|\eta|>0$, then $T-\lambda I-\eta I$ is a Weyl operator. As $\lambda \in E_{a}(T)$, then if $\eta$ is chosen small 
enough, we would have $\alpha(T-\lambda I-\eta I)=0$. As $T-\lambda I-\eta I$ is a Weyl operator, then $\beta(T-\lambda I-\eta I)=0$, and $T-\lambda I-\eta I$ is invertible. But this is a contradiction, since $\lambda \notin E(T)$. Consequently, $\lambda \in \sigma_{B W}(T)$, and $\sigma_{B W}(T) \backslash \sigma_{S B F_{+}^{-}}(T)=$ $\left(\sigma(T) \backslash \sigma_{a}(T)\right) \bigsqcup\left(E_{a}(T) \backslash E(T)\right)$. As $\sigma(T)=\sigma_{a}(T) \bigsqcup\left(\sigma(T) \backslash \sigma_{a}(T)\right.$, then

$$
\begin{aligned}
\sigma(T) & =\sigma_{S B F_{+}^{-}}(T) \bigsqcup E_{a}(T) \bigsqcup\left(\sigma(T) \backslash \sigma_{a}(T)\right. \\
& =\sigma_{S B F_{+}^{-}}(T) \bigsqcup E(T) \bigsqcup\left(E_{a}(T) \backslash E(T)\right) \bigsqcup\left(\sigma(T) \backslash \sigma_{a}(T)\right. \\
& =\sigma_{S B F_{+}^{-}}(T) \bigsqcup E(T) \bigsqcup\left(\sigma_{B W}(T) \backslash \sigma_{S B F_{+}^{-}}(T)\right) \\
& =\sigma_{B W}(T) \bigsqcup E(T) .
\end{aligned}
$$

Therefore $T$ satisfies generalized Weyl's theorem.

Conversely, assume that $T$ satisfies generalized Weyl's theorem, and

$$
\sigma_{B W}(T) \backslash \sigma_{S B F_{+}^{-}}(T)=\left(\sigma(T) \backslash \sigma_{a}(T)\right) \bigsqcup\left(E_{a}(T) \backslash E(T)\right) .
$$

Then

$$
\begin{aligned}
\sigma(T) & =\sigma_{a}(T) \bigsqcup\left(\sigma(T) \backslash \sigma_{a}(T)\right. \\
& =\sigma_{B W}(T) \bigsqcup E(T) \\
& =\left(\sigma_{B W}(T) \backslash \sigma_{S B F_{+}^{-}}(T)\right) \bigsqcup \sigma_{S B F_{+}^{-}}(T) \bigsqcup E(T) \\
& =\left(\sigma(T) \backslash \sigma_{a}(T)\right) \bigsqcup\left(E_{a}(T) \backslash E(T)\right) \bigsqcup \sigma_{S B F_{+}^{-}}(T) \bigsqcup E(T) \\
& =\left(\sigma(T) \backslash \sigma_{a}(T)\right) \bigsqcup \sigma_{S B F_{+}^{-}}(T) \bigsqcup E_{a}(T) .
\end{aligned}
$$

Hence $\sigma_{a}(T)=\sigma_{S B F_{+}^{-}}(T) \bigsqcup E_{a}(T)$, and $T$ satisfies generalized a-Weyl's theorem.

In the following example, we define an operator $T$ which satisfies generalized Weyl's theorem and property $\left(U W_{E}\right)$, but $T$ does not satisfies generalized aWeyl's theorem, nor property $\left(U W E_{a}\right)$.

Example 3.10. Let $T$ be the operator defined on the Banach space $\ell^{2}(\mathbb{N}) \oplus$ $\ell^{2}(\mathbb{N}) \oplus \ell^{1}(\mathbb{N})$ by $T=R \oplus 0 \oplus Q$, where $R$ is the unilateral right shift operator and $Q$ the operator defined by $x=\left(\xi_{i}\right) \in l^{1}$ by:

$$
Q\left(\xi_{1}, \xi_{2}, \xi_{3}, \ldots, \xi_{k}, \ldots\right)=\left(0, \alpha_{1} \xi_{1}, \alpha_{2} \xi_{2}, \ldots, \alpha_{k-1} \xi_{k-1}, \ldots\right),
$$

where $\left(\alpha_{i}\right)$ is a sequence of complex numbers such that $0<\left|\alpha_{i}\right| \leq 1$ and $\sum_{i=1}^{\infty}\left|\alpha_{i}\right|<\infty$. We observe that $\overline{R\left(Q^{n}\right)} \neq R\left(Q^{n}\right), n=1,2, \ldots$ Indeed, for a given $n \in \mathbb{N}$ let $x_{k}^{(n)}=(1, \ldots, 1,0,0, \ldots)$ (with $n+k$ times 1$)$. Then the limit $y^{(n)}=\lim _{k \rightarrow \infty} Q^{n} x_{k}^{(n)}$ exists and lies in $\overline{R\left(Q^{n}\right)}$. However, there is no element $x^{(n)} \in \ell^{1}$ satisfying the equation $Q^{n} x^{(n)}=y^{(n)}$ as the algebraic solution to this equation is $(1,1,1, \ldots) \notin \ell^{1}$. 
We have $\sigma_{a}(T)=\sigma_{S F_{+}^{-}}(T)=C(0,1) \cup\{0\}, \sigma_{S B F_{+}^{-}}(T)=C(0,1) \cup\{0\}$ and $E_{a}(T)=\{0\}$. Moreover, we have $\sigma(T)=\sigma_{W}(T)=\sigma_{B W}(T)=D(0,1)$ and $E(T)=\emptyset$. So $T$ satisfies generalized Weyl's theorem, but $T$ does not satisfies generalized a-Weyl's theorem. We note that $\sigma_{B W}(T) \backslash \sigma_{S B F_{+}^{-}}(T) \neq$ $\left(\sigma(T) \backslash \sigma_{a}(T)\right) \bigsqcup\left(E_{a}(T) \backslash E(T)\right)$.

In [11, Theorem 3.8] it had been proved that condition (i) of the following theorem implies the first part of its second condition (ii). Here, we obtain an equivalence of the statements (i) and (ii).

Theorem 3.11. Suppose that $T \in L(X)$. Then the following statements are equivalent:

(i) T satisfies generalized a-Browder's theorem;

(ii) $T$ satisfies generalized Browder's theorem, and $\sigma_{B W}(T) \backslash \sigma_{S B F_{+}^{-}}(T)=$ $\left(\sigma(T) \backslash \sigma_{a}(T)\right) \bigsqcup\left(\Pi_{a}(T) \backslash \Pi(T)\right)$.

In [18, Corollary 2.5] it had been proved that condition (i) of the following theorem implies the first part of its second condition (ii). Here, we obtain an equivalence of the statements (i) and (ii).

Theorem 3.12. Suppose that $T \in L(X)$. Then the following statements are equivalent:

(i) T satisfies a-Weyl's theorem;

(ii) $T$ satisfies Weyl's theorem, and

$$
\sigma_{W}(T) \backslash \sigma_{S F_{+}^{-}}(T)=\left(\sigma(T) \backslash \sigma_{a}(T)\right) \bigsqcup\left(E_{a}^{0}(T) \backslash E^{0}(T)\right) .
$$

In [14, Theorem 3.9] it had been proved that condition (i) of the following theorem implies the first part of its second condition (ii). Here, we obtain an equivalence of the statements (i) and (ii).

Theorem 3.13. Suppose that $T \in L(X)$. Then the following statements are equivalent:

(i) T satisfies a-Browder's theorem;

(ii) T satisfies Browder's theorem, and

$$
\sigma_{W}(T) \backslash \sigma_{S F_{+}^{-}}(T)=\left(\sigma(T) \backslash \sigma_{a}(T)\right) \bigsqcup\left(\Pi_{a}^{0}(T) \backslash \Pi^{0}(T)\right) .
$$

In $[1$, Theorem 2.3] it had been proved that condition (i) of the following theorem implies the first part of its second condition (ii). Here, we obtain an equivalence of the statements (i) and (ii).

Theorem 3.14. Let $T \in L(X)$. Then the following statements are equivalent:

(i) $T$ satisfies property $(\mathrm{gw})$;

(ii) $T$ satisfies property $(w)$, and $\sigma_{S F_{+}^{-}}(T) \backslash \sigma_{S B F_{+}^{-}}(T)=E(T) \backslash E^{0}(T)$. 
In [13, Theorem 2.3] it had been proved that condition (i) of the following theorem implies the first part of its second condition (ii). Here, we obtain an equivalence of the statements (i) and (ii).

Theorem 3.15. Let $T \in L(X)$. Then the following statements are equivalent:

(i) $T$ satisfies property $(g b)$;

(ii) $T$ satisfies property $(b)$, and $\sigma_{S F_{+}^{-}}(T) \backslash \sigma_{S B F_{+}^{-}}(T)=\Pi(T) \backslash \Pi^{0}(T)$.

\section{References}

[1] M. Amouch and M. Berkani, On the property (gw), Mediterr. J. Math. 3 (2008), no. 3, $371-378$.

[2] M. Berkani, On a class of quasi-Fredholm operators, Integral Equations Operator Theory 34 (1999), no. 2, 244-249.

[3] - Restriction of an operator to the range of its powers, Studia Math. 140 (2000), no. $2,163-175$.

[4] _ Index of B-Fredholm operators and generalization of a Weyl theorem, Proc. Amer. Math. Soc. 130 (2002), no. 6, 1717-1723.

[5] _ B-Weyl spectrum and poles of the resolvent, J. Math. Anal. Appl. 272 (2002), no. $2,596-603$.

[6] _ On the equivalence of Weyl theorem and generalized Weyl theorem, Acta Math. Sin. (Engl. Ser.) 23 (2007), no. 1, 103-110.

[7] M. Berkani and A. Arroud, Generalized Weyl's theorem and hyponormal operators, J. Aust. Math. Soc. 76 (2004), no. 2, 291-1302.

[8] M. Berkani, N. Castro, and S. V. Djordjević, Single valued extension property and generalized Weyl's theorem, Math. Bohem. 131 (2006), no. 1, 29-38.

[9] M. Berkani and M. Kachad, New Weyl-type Theorems. I, Funct. Anal. Approx. Comput. 4 (2012), no. 2, 41-47.

[10] M. Berkani, M. Kachad, H. Zariouh, and H. Zguitti, Variations on a-Browder's Theorem, Sarajevo. J. Math. 9 (2013), no. 2, 271-281.

[11] M. Berkani and J. J. Koliha, Weyl type theorems for bounded linear operators, Acta Sci. Math. (Szeged) 69 (2003), no. 1-2, 359-376.

[12] M. Berkani and M. Sarih, On semi B-Fredholm operators, Glasg. Math. J. 43 (2001), no. 3, 457-465.

[13] M. Berkani and H. Zariouh, Extended Weyl type theorems, Math. Bohem. 134 (2009), no. $4,369-378$.

[14] S. V. Djordjević and Y. M. Han, Browder's theorems and spectral continuity, Glasg. Math. J. 42 (2000), 479-486.

[15] H. Heuser, Functional Analysis, John Wiley \& Sons Inc, New York, 1982.

[16] S. Grabiner, Uniform ascent and descent of bounded operators, J. Math. Soc. Japan 34 (1982), no. 2, 317-337.

[17] K. B. Laursen and M. M. Neumann, An introduction to Local Spectral Theory, Clarendon Press Oxford, 2000.

[18] V. Rakočević, Operators obeying a-Weyl's theorem, Rev. Roumaine Math. Pures Appl. 34 (1989), no. 10, 915-919.

[19] H. Weyl, Über beschränkte quadratische Formen, deren Differenz vollstetig ist, Rend. Circ. Mat. Palermo 27 (1909), 373-392.

[20] H. Zariouh and H. Zguitti, Variations on Browder's theorem, Acta Math. Univ. Comenian. 81 (2012), no. 2, 255-264. 
MOHAMMED BERKANI

DEPARTMENT OF MATHEMATICS

Operator Theory Team

SCIENCE FACUlTy OF OUjdA

University Mohammed I, Morocco

E-mail address: berkanimo@aim.com

MOHAMMED KACHAD

DEPARTMENT OF MATHEMATICS

Operator Theory Team

SCIEnCE FACUlty of Oujda

University Mohammed I, Morocco

E-mail address: kachad.mohammed@gmail.com 